\title{
A New Species and Record of Aceria (Acari: Prostigmata: Eriophyoidea) on Weeds from Egypt
}

\author{
Elhalawany, A. S.; K. M. El-Sayed and A. I. Amer \\ Plant Protection Research Institute, ARC, Dokki, Giza, Egypt, dr_ashraf_said@yahoo.com.
}

\begin{abstract}
Eriophyoids are of great economic importance as pests of weeds, particularly in causing sometimes deformities such as bud galls, stunting, rusting, bronzing, leaf rolling, erineum, blisters, galls, coating, damaged seeds and mosaic virus disease. During the survey of eriophyoid mite fauna from Qualyubia, Cairo and Gharbia governorates in Egypt from 2014 to 2018. One new species and one new record of the genus Aceria from weeds are described and illustrated. Aceria lividus sp. nov., was collected from Amaranthus lividus L. (Amaranthaceae), vagrant on buds and flowers causing stunt and deformation. This species is the first record of eriophyoid mite from the family Amaranthaceae from Egypt. A supplementary description of the first record of Aceria malherbae Nuzzaci, 1985 infested Convolvulus arvensis L. (Convolvulaceae) in Egypt based on females, males and immature. This mite found on upper leaf surface alongside the midrib, causes leaf folding, deformation of flowers and plants and prevent plant reproduction. The two Aceria species indicate that is a promising candidate for the biological control of $C$. lividus and $C$. arvensis.
\end{abstract}

Key words: Eriophyidae, Aceria, Amaranthus lividus, Convolvulus arvensis, weeds, taxonomy.

\section{INTRODUCTION}

Eriophyoidea are an economically important group because the direct damage they can cause to their hosts, their ability to transmit serious plant diseases, and also due to the possibility of using them as biological agents for weed control (Lindquist et al., 1996). Nearly $80 \%$ have been reported on a single host species, $95 \%$ on one host genus and $99 \%$ on one host family (Skoracka et al., 2010).

Amaranthus is a cosmopolitan genus compresses almost 65 species, distributed in the tropical, subtropical and warm region of the world (Boulos, 1999). It is a serious problem weed in several field crops as well as in vegetables and orchards. It is an annual spreading by seeds which have a long viability and are dispersed principally by wind and water, but also by machinery (Zaki, 2000).

Along with the current new records, more than 28 named eriophyoid species have been collected and described from plants of the family Amaranthaceae, 20 of them belong to genus Aceria and none of them has been found in Egypt. Only two Aceria species collected up to now from Amaranthus: Aceria amaranthi Abou-Awad \& El-Banhawy, 1992 collected from numerous galls on both leaf surfaces, causing malformation of the leaves of Amaranthus sp. from Tanzania and Aceria vanensis Denizhan \& Kiedrowicz, 2016 vagrant on leaves of Amaranthus retroflexus L. in Turkey (Zaher 1984; Abou-Awad \& El-Banhawy, 1992; Elhalawany 2012; Denizhan et al., 2016 \& Amrine and de Lillo personal communication) Table 1.

Field bindweed, Convolvulus arvensis L. (Convolvulaceae) is an aggressive perennial weed of
Mediterranean and Middle East origin, found throughout temperate regions of the world. It is distributed from 44 countries and in 2 different crops, and it's ranked as the $12^{\text {th }}$ most important weed in the world (Holm et al., 1977). Field bindweed's long lived seeds and extensive root system make it extremely difficult to control by chemical and mechanical means (Rosenthal, 1983).

Aceria malherbae Nuzzaci, 1985 was recorded on Convolvulus arvensis L. from Italy, on leaf feeding starts on the upper surface alongside the midrib, usually towards the distal end of the leaf. Galled leaves become yellow-green or red. When mites attack the bud they also prevent natural stem growth and elongation (Rosenthal, 1996). A. malherbae was release against $C$. arvensis in the USA during 1987 (Rosenthal \& Platta, 1990), and in South Africa in 1995 (Creamer, 1995) and established in Texas, but the effect on the weeds is not yet known (Boldt \& Sobhian, 1993).

The knowledge on eriophyoid mite fauna of Egypt is limited. Reviewing literature showed that up to 2012 only 65 eriophyoid species have been discovered and described from Egypt. Since then more eriophyoids have been recorded and described by several authors (e.g., Zaher 1984, Elhalawany, 2012, 2014a, 2014b, 2015, 2017). As a result of these studies, the list of eriophyoid mites recorded in Egypt has reached up to 101 species belonging to 32 genera. More than 1,000 named species have been assigned to the genus Aceria Keifer, 1944 and about 38 of them have been found in Egypt up to now (Elhalawany \& Ueckermann 2015\& 2018).

The current contribution is aimed to description of a new eriophyoid mite species, namely Aceria lividus 
Table 1. Eriophyoid mite species collected from Amaranthaceae plants worldwide and their type host, habits and, type locality.

\begin{tabular}{|c|c|c|c|}
\hline Species & Type host & Habits & type locality \\
\hline Aceria heimi (Nalepa, 1899a) & Atriplex halimus L. & $\begin{array}{l}\text { flower deformation and erineum, } \\
\text { halophytic }\end{array}$ & France \\
\hline Aceria brevipes (Nalepa, 1899c) & Atriplex halimus L. & $\begin{array}{l}\text { hemispherical indentations in the } \\
\text { leaf surface }\end{array}$ & Italy \\
\hline Aceria caulobia (Nalepa, 1900) & Suaeda vera J. F. Gmelin in L. & galls & Italy \\
\hline Aceria salicorniae (Nalepa, 1902) & Arthrocnemum fruticosum (L.) Moq. & witches' broom & Cyprus \\
\hline Aceria obiones (Molliard, 1904) & Atriplex pedunculata $\mathrm{L}$. & flower stalk galls & France \\
\hline Aceria allenrolfeae Keifer, 1952 & $\begin{array}{l}\text { Allenrolfea occidentalis } \quad \text { (Wats.) } \\
\text { Kuntze. }\end{array}$ & $\begin{array}{l}\text { the mites inhabit joints and flowers } \\
\text { heads }\end{array}$ & USA \\
\hline Aceria rubifaciens Lamb, 1953 & Salicornia australis Sol. ex Forst. f. & galls on shoot & New Zealand \\
\hline Aceria desertorum Roivainen, 1953 & Suaeda vera J. F. Gmelin in L. & vagrant & Spain \\
\hline Aceria sarcobati Keifer, 1962 & Sarcobatus vermiculatus (Hook.) Torr. & produce blister-like leaf swellings & USA \\
\hline Aceria atriplicis Wilson \& Oldfield, 1966 & Atriplex polycarpa (Torr.) S. Watson & leaf bead galls & USA \\
\hline Aceria beniciae Keifer, 1966 & Salicornia virginica $\mathrm{L}$. & the mites live between stem joints & USA \\
\hline Aceria achyranthi Mohanasundaram, 1990 & Achyranthes aspera $\mathrm{L}$. & $\begin{array}{l}\text { undersurface leaf vagrant, slight } \\
\text { rusting. }\end{array}$ & India \\
\hline Aceria aervae Mohanasundaram, 1990 & Aerva lanata (L.) Juss. ex Schult. & vagrant & India \\
\hline $\begin{array}{llll}\text { Aceria amaranthi } & \text { Abou-Awad } & \& & \text { El- } \\
\text { Banhawy, } 1992 & & & \end{array}$ & Amaranthus sp. & $\begin{array}{l}\text { numerous galls on both leaf surface, } \\
\text { causing malformation of the leaves }\end{array}$ & Tanzania \\
\hline Aceria zumetae Boczek \& Petanovic, 1994 & Krascheninnikovia ceratoides (L.) & causing small galls on leaves & Spain \\
\hline Aceria salsolae de Lillo, 1996 & Salsola kali L. & $\begin{array}{l}\text { infested plants remain stunted and } \\
\text { less spiny; seed production is } \\
\text { drammatically reduced }\end{array}$ & Turkey \\
\hline $\begin{array}{l}\text { Aceria chenopodia Xue, Sadeghi \& Hong, } \\
2009\end{array}$ & Chenopodium lividus L. & vagrant on flowering parts & Iran \\
\hline Aceria vanensis Denizhan \& Kiedrowicz, 2016 & Amaranthus retroflexus $\mathrm{L}$. & vagrant & Turkey \\
\hline Aceria halocnemi Lotfollahi \& Tajaddod, 2018 & $\begin{array}{l}\text { Halocnemum strobilaceum (Pall.) } \mathrm{M} . \\
\text { Bieb. }\end{array}$ & vagrant & Iran \\
\hline Aceria lividus Elhalawany sp. nov. & Amaranthus lividus L. (A. blitum) & $\begin{array}{l}\text { vagrant on buds and flowers causes } \\
\text { stunted, deformed. }\end{array}$ & Egypt \\
\hline
\end{tabular}

sp. nov., infested $C$. lividus in Egypt. It also supplementary description and illustration of immature stages of $A$. malherbae infested $C$. arvensis as a new science for the Egyptian fauna.

\section{MATERIALS AND METHODS}

During the five year period from 2014-2018, random samplings of weeds showing symptoms of mite infestation were carried out in Qualyubia, Cairo and Gharbia governorates in Egypt. Eriophyoid mites were collected from the plant samples by direct examination with the aid of a stereo-microscope and mounted on microscope slides in Keifer's F-medium (Amrine and Manson 1996). The specimens were examined under a phase contrast microscope (Carl Ziess Nr, German). Illustrations were made with the use of drawing tube attached to the phase contrast microscope and using the Adobe Illustrator ${ }^{\circledR}$ CS6 program. Identifications to genus level was conducted using a published key to the world genera of the Eriophyoidea (Amrine et al., 2003). Morphological terminology is based on Lindquist (1996) and data measurements follow (Amrine and Manson 1996; de Lillo et al., 2010). All measurements were made using the software computer program (compu Eye) (Baker, 2005) and are given in micrometres $(\mu \mathrm{m})$ and the number of measured specimens (n) is given within parentheses in the description. For males and immature stages, only the ranges are given.

\section{RESULTS AND DISCUSSION}

\author{
Family Eriophyidae Nalepa, 1898 \\ Subfamily Eriophyinae Nalepa, 1898 \\ Tribe Aceriini Amrine and Stasny, 1994 \\ Aceria lividus Elhalawany sp. nov. (Figures 1-4)
}

\section{Description}

FEMALE: (n=15) Body vermiform, 200 (173-213) long without gnathosoma, 52 (50-62) wide, 51 (46-62) thick; whitish in life. Gnathosoma 25 (25-28) long, projecting obliquely downwards, basal setae ep 3 (2-3), antapical setae $d 6$ (5-6), chelicerae 17 (16-18) long. Prodorsal shield 34 (33-35) long with short frontal lobe acuminate, 45 (42-50) wide; semicircular; prodorsal shield ornamentation with median line complete, broken basal one-third; admedian lines complete, gently diverging to rear, first submedian line from side of admedian at anterior edge ending at $1 / 2$; lateral line from anterior end of submedian soon becoming granular; numerous granules and short dashes in area between median and admedian lines, and lateral sides of prodorsal shield. Scapular tubercles on rear shield margin, 30 (26-30) apart, setae sc 26 (24-27), projecting posteriorly. Coxigenital area with granules, with 5 (5-6) annuli between coxae and genitalia, prosternal apodeme present 6 (5-7); anterolateral setae on coxisternum 
I $1 b 7$ (7-9), 14 (14-15) apart; proximal setae on coxisternum I la 23 (22-25), 12 (12-13) apart; proximal setae on coxisternum II $2 a 27$ (25-33), 28 (27-30) apart. Leg I 33 (30-35), femur10 (10-11), basiventral femoral setae $b v 10$ (10-12); genua 5 (5-6), antaxial genual setae $l^{\prime \prime} 25$ (24-27); tibiae 8 (7-9), paraxial tibial setae $l^{\prime} 6$ (6-7), setae located 1/4 from dorsal base; tarsi 7 (6-7); empodia em simple 5 (4-5), 6-rayed, tarsi solenidia $\omega$ slightly tapered, 8 (7-9), paraxial fastigial tarsal setae $f^{\prime} 15$ (14-17), antaxial fastigial tarsal setae $f t^{\prime \prime} 25$ (24-27), tarsal setae $u^{\prime} 2-3$. Leg II 29 (28-32), femur 10 (10-11), setae bv $11 \quad(10-12)$; genua 5 (4-5), setae $l^{\prime \prime} 10$ (10-12); tibiae 7 (6-8); tarsi 6 (6-7); tarsal em simple 5 (4-5), 6-rayed, w slightly tapered 8 (7-9), setae $f t^{\prime} 8$ (7-10), setae $f t^{\prime \prime} 22(20-25)$, tarsal setae $u^{\prime} 2-3$. Opisthosoma with 65 (63-68) dorsoventrally annuli subequal, with elliptical microtubercles on rear annular margins; ventrally with round microtubercles on rear annular margins, the last $10^{\text {th }}$ ventral microtubercles liner. Lateral setae $c 225$ (25-27), 48 (47-50) apart, on annulus 9 (9-10) from coxae II; ventral setae I $d 55$ (53-60), 41 (30-43) apart, on annulus 21 (20-22); ventral setae II $e$ 11 (9-12), 21 (20-22) apart, on annulus 34 (33-35); ventral setae III $f 25$ (24-30), 23 (22-23) apart, on $7^{\text {th }}$ annulus from rear. Setae h2 65 (64-70); setae $h 13$ (3-4). External genitalia 16 (15-18), 23 (22-25) wide, coverflap with 12-14 longitudinal ridges in a single row, proximal setae on coxisternum III $3 a, \quad 17 \quad(15-18)$, 18 (18-19) apart.

MALE. $(\mathrm{n}=10)$. Similar to female. Body vermiform, 150-165 including gnathosoma, 46-53 wide, 45-48 thick; whitish in life. Gnathosoma 22-25, chelicerae 15-17, setae ep 3-4, setae $d$ 5-6. Prodorsal shield shape and patterns similar to those of the female, 28-30 long including frontal lobe, 42-45 wide; Scapular tubercles near the rear shield margin, 24-26 apart, setae sc 16-19, projecting diagonal posteriorly. Coxigenital area with granules, prosternal apodeme present 5-6; setae $1 b$ 5-6, 11-12 apart; setae $1 a$ 12-14, 10-11 apart; setae $2 a$ 24-27, 20-21 apart. Leg I 26-29, femora 8-9, setae bv 9-11; genu 5-6, setae $l^{\prime \prime} 17-19$; tibiae 6-7, setae $l^{\prime} 4-5$; tarsi 7-8; tarsal empodia em simple 5-6, 6 -rayed, tarsal solenidia $\omega$ distally slight tapered 6-8, setae $f t^{\prime} 11-13$, setae $f t^{\prime \prime} 17-20$, setae $u^{\prime} 2-3$. Leg II 24-27, femora 7-8, setae bv 9-10; genu 4-5, setae $l^{\prime \prime}$ 7-9; tibiae 5-6; tarsi 6-7; tarsal empodia em simple 5-6, 6-rayed, tarsal solenidia $\omega$ distally slight tapered 5-6, setae $f t^{\prime} 5-7$, setae $f t^{\prime \prime} 17-22$, setae $u^{\prime} 2$ 3. Opisthosoma with 55-60 dorsoventrally annuli subequal, microtubercles shape same that of the female. Lateral setae $c 2$ 17-22, 48-50 apart, on annulus 9 from coxae II; ventral setae $d$ 38-40,
[33-37 apart, on annulus 18-20; setae $e$ 8-10, 18-19 apart, on annulus 30-32; setae $f 20-24,18-20$ apart, on $7^{\text {th }}$ annulus from rear. Setae $h 244-55$; setae h1 3-4. External genitalia 12-13 long, 19-20 wide, with granules, setae $3 a 12-15,16-17$ apart.

Nymph (n=6). Body vermiform, 150-167; width 40-43. Gnathosoma 20-23, curved downward, setae $d$ 4-5, ep 2-3, chelicerae 16-18. Prodorsal shield sub-circular, 39-42, including frontal lobe 4-6 over the gnathosomal base 40-42 wide, with pattern similar to that of female. Tubercles $s c$ on rear shield margin, 20-21 apart; sc 14-17. Coxisternal plates With granules, $1 b$ 5-6, 11-12 apart; la 13-15, 8-9 apart; $2 a$ 22-25, 25-26 apart; $3 a$ 9-10, 9-10 apart. Leg I 24-26; femur 7-8, bv 8-10; genu 4-5, $l^{\prime \prime}$ 12-15; tibia 5-6, $l^{\prime}$ 4-5; tarsus 4-5, ft' 11-13, $f^{\prime \prime}$ 15-17, setae $u^{\prime} 2$; solenidion $\omega 5-6$; em 4, simple, 5-rayed. Leg II 22-24; femur 6-7, bv 8-10; genu 3-4, l' 5-8; tibia 4-5; tarsus 4-5, ft' 7-8, $f^{\prime \prime} 12-14$, setae $u^{\prime} 2 ; \omega 5$ 5-6; em 4-5, simple, 5-rayed. Opisthosoma With 52-56 dorsal annuli, with elliptical microtubercles situated on rear margin of each semiannulus, 46-50 ventral annuli with minute round microtubercles, situated on rear margin of each semiannulus; elongated on the posterior annuli. Setae $c 2$ 21-23, 40-42 apart, on 8-9 ventral semiannulus; setae $d$ 30-35, 28-30 apart, on 18-19 ventral semiannulus; setae $e$ 12-14, 17-18 apart, on 27-28 ventral semiannulus; setae $f 20-23,19-20$ apart, on $6^{\text {th }}$ semiannulus from rear. Setae $h 12-3 ; h 2$ 30-44.

LARVA ( $\mathrm{n}=4)$. Body vermiform, 142-155; width 38-40. Gnathosoma 20-22 curved downward, setae d 3-4, ep 1-2, cheliceral stylets 15-16. Prodorsal shield sub-circular, 24-26, including frontal lobe 2-3 over the gnathosomal base 24-27 wide; median and sub- median complete; broken, admedian lines from anterior $1 / 2$, subparallel; granules present on lateral sides of prodorsal shield. Tubercles $s c$ on rear shield margin, 18-20 apart; setae sc 14-17. Coxisternal plates With granules setae $1 b$ 5-6, 9-10 apart; $1 a$ 11-13, 7-8 apart; 2a 17-20, 19-21 apart. setae 3a 4-5, 6-7 apart. Leg I 23-25; femur 5-6, bv 5-6; genu 3-4, $l^{\prime \prime} 13-15$; tibia 3-4, seta $l^{\prime} 3-$ 4; tarsus 5-6, ft' 9-10, ft" 14-15; $\omega$ 5-6; em 3-4, simple, 4-rayed. Leg II 18-20; femur 5-6, bv 5-6; genu 3, l" 5-7; tibia 3-3.5; tarsus 4-5, ft' 4-5, $f^{\prime \prime}$ 14-17; $\omega$ 5-6; em 3-4, 4-rayed. Opisthosoma With subequal annuli, 30-36 annuli, with minute round microtubercles situated on rear margin of each semiannulus. Seta $c 2$ 17-20, 35-37 apart, on 9 ventral semiannulus; setae $d$ 19-23, 19-20 apart, on 14 ventral semiannulus; setae $e 7-8,12-13$ apart, on 20 ventral semiannulus; setae $f 16-17,14-15$ apart, on $6^{\text {th }}$ semiannulus from rear; $h 11-2 ; h 2$ 17-22. 


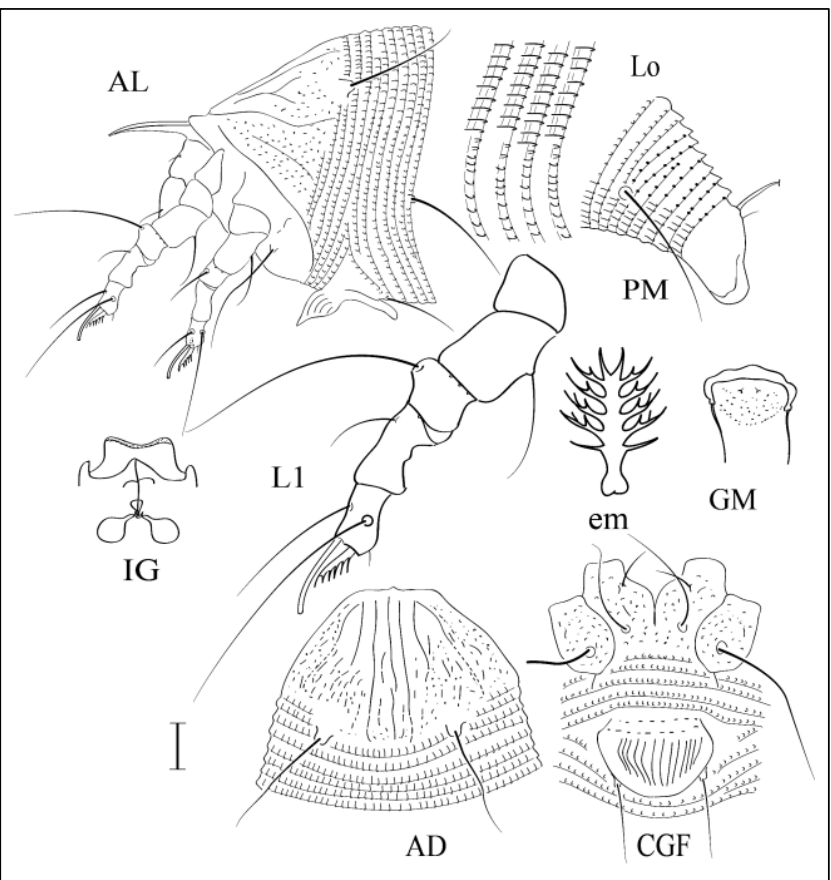

Fig. (1): Line drawings of Aceria lividus sp. nov.: AD- prodorsal shield of female; CGF- female coxigenital region; GM- male genitalia; IGinternal female genitalia; em- empodium; L1Leg I; AL- anterio-lateral view of mite; Lolateral view of annuli; PM- posterior lateral view of mite. Scale bars: $10 \mu \mathrm{m}$ for AD, CGF, GM, IG, $\mathrm{Al}, \mathrm{PM} ; 5 \mu \mathrm{m}$ for $\mathrm{L} 1 ; 2.5 \mu \mathrm{m}$ for em.

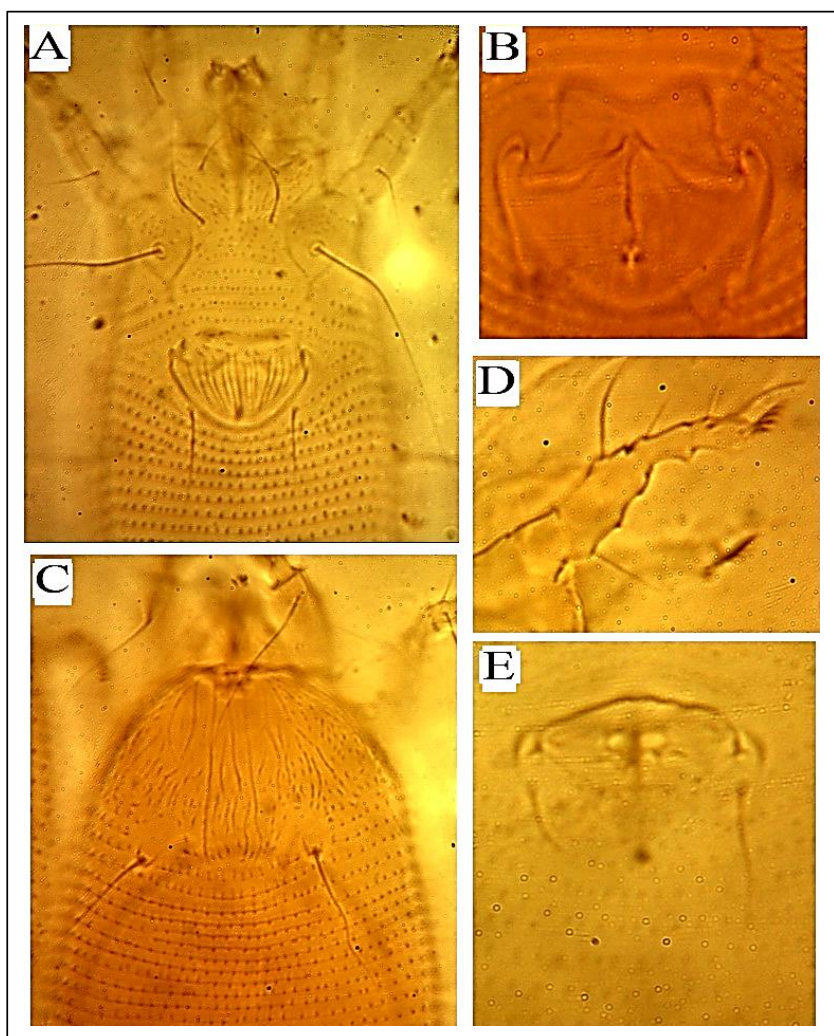

Fig. (3): Phase microphotograph of Aceria lividus sp. nov.: A- female coxigenital region; $\mathrm{B}$ - internal female genitalia; $\mathrm{C}$ - prodorsal shield of female; D- Leg I; E- male genitalia.

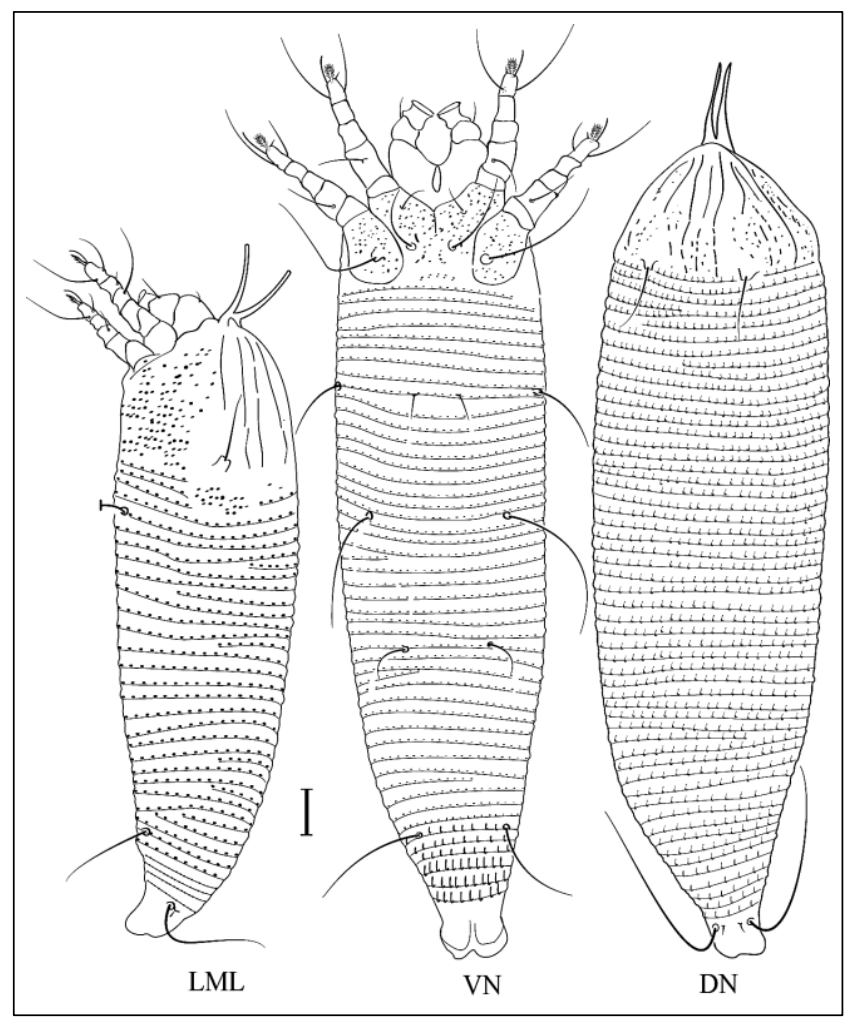

Fig. (2): Line drawings of Aceria lividus sp. nov.: $\mathrm{DN}$ - dorsal view of nymph; $\mathrm{VN}$ - ventral view of nymph; LML- lateral view of larva. Scale bars: $10 \mu \mathrm{m}$.

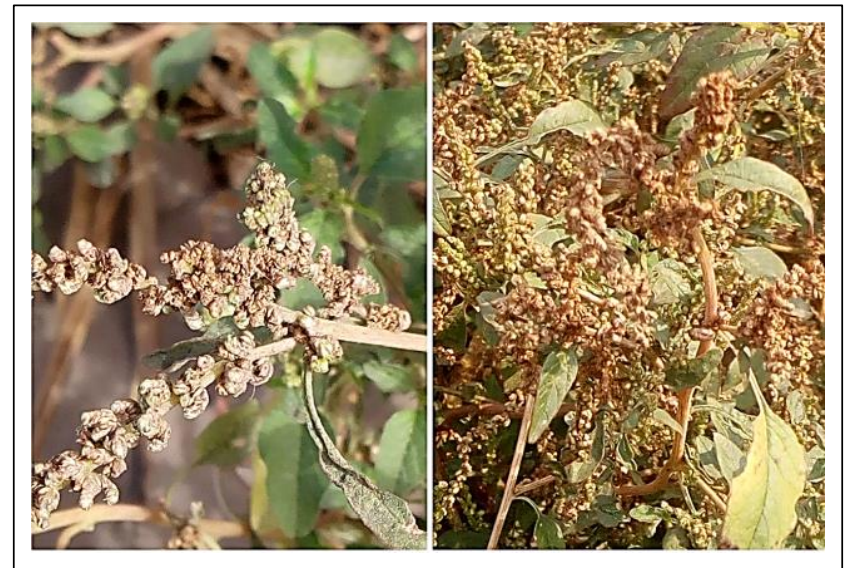

Fig. (4): Plant damage caused by eriophyoid mite Aceria lividus sp. nov. 
Host plant. Amaranthus lividus L. = (Amaranthus blitum L.) (Amaranthaceae).

Relation to the host plant. Vagrant on buds and flowers causes stunted, deformed (Figure 4).

Type Locality. El-Sad village, Qalyubia

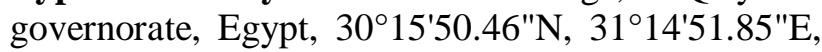
15 October 2015 and 17 September 2018 coll. Ashraf S. Elhalawany. New Cairo, 20 October 2018 coll Karam M. El-Sayed. Kafr Ibri, Gharbia governorate, $30^{\circ} 41^{\prime} 56.00 " \mathrm{E}, 31^{\circ} 10^{\prime} 49.07 " \mathrm{~N}, 10$ September 2018 coll. Ahmad Amer.

Type material. Holotype, single female on a microscope slide (slide no. EGPErio62.1), deposited in Fruit Trees Mites Dept., Collection, Plant Protection Research Institute (PPRI), Dokki, Egypt. Paratypes: 25 females and 10 males 6 nymph paratypes and 5 larva paratypes on 10 separate microscope slides deposited in Fruit Trees Mites Dept., Collection, Plant Protection Research Institute (PPRI), Dokki, Egypt. Two slides in Plant Protection Research Institute collection. Two paratype slides are deposited at Plant Protection Research Institute collection. Two paratypes slide mounts are also deposited in the Department of Soil, Plant and Food Sciences (Di.S.S.P.A.), section of Entomology and Zoology, University of Bari Aldo Moro, Bari, Italy.

Etymology. The species name is based on the host plant species name lividus .

Differential diagnosis. The new species herein described was compared with all Aceria species found on Amaranthaceaus plants and no similarities among any of them were observed. Aceria lividus sp. nov. is slightly related to Aceria amaranthi Abou-Awad \& El-Banhawy, 1992 collected from numerous galls on both leaf surfaces, causing malformation of the leaves of Amaranthus sp. from Tanzania and Aceria vanensis Denizhan \& Kiedrowicz, 2016 vagrant on leaves of Amaranthus retroflexus L. in Turkey. The new species distinguished from A. amaranthi by the length of setae $c 2, d$, and $f$, shape of dorsal microtubercles, tarsal empodium and ornamentation of prodorsal shield. Females of the newly described species have granules and short dashes in area between median and admedian lines, and lateral sides of prodorsal shield, with elliptical microtubercles on dorsal annuli, tarsal emopidum 6-rayed, longest length of $c 2, d$, and $f(27-27,5-60$ and 24-0, respectively). In the female of $A$. amaranthi without granules on prodorsal shield, with rounded microtubercles, tarsal empodium 5-rayed, shortest length of $c 2, d$, and $f(11,37$ and 16 , respectively).
Also, the new species differs from A. vanensis by prodorsal shield design, length of $e$ and $3 a$ setae, shape of dorsal microtubercles and number of longitudinal ridges on genitalia, and coxae ornamentation. Prodorsal shield without median line and submedian lines; admedian lines with short lines and without granules in A. vanensis (versus median, admedian lines complete and short submedian lines and shield with granules and short dashes in the new species); longer $e$ setae $45-55 \mu$ and $3 a 21-25$ long in A. vanensis (versus 9-12 and 15-18 in the new species); with rounded microtubercles dorsally in the new species(versus elliptical in the new species); genital coverflap smooth in A. vanensis (versus 12 14 longitudinal ridges in new species), and coxae sooth in A. vanensis versus with granules and short lines in the new species.

\section{Aceria malherbae Nuzzaci, 1985. First record (Figs} 5-8).

Aceria malherbae Nuzzaci, 1985: 81-89.

Aceria malherbae; Creamer 1993: 53-95.

Aceria malherbae; Amrine \& Stasny, 1994: 62.

Aceria malherbae; Baker et al., 1996: 114.

Aceria malherbae; Xue et al., 2013: 45-47.

\section{Redescription}

FEMALE: (n=15) Body vermiform, 200 (180-245) long without gnathosoma, 64 (60-69) wide, 64 (63-67) thick; whitish in life. Gnathosoma 25 (24-30) long, projecting obliquely downwards, basal setae ep 3 (2-3), antapical setae $d 6$ (6-7), chelicerae 17 (15-18) long. Prodorsal shield 44 (41-46) long with short frontal lobe acuminate, 52 (51-53) wide; semicircular; anterior shield lobe somewhat acuminate; median line indistinct on rear of prodorsal shield forming V-shaped marks, admedian lines incomplete and present only on the posterior half to two third of the prodorsal shield, submedian line I faint present on basal $1 / 2$, curved inwards laterally to reach up to the middle of the prodorsal shield; submedian line II present on the posterior $2 / 3$ on lateral side. Scapular tubercles on rear shield margin, 31 (30-34) apart, setae sc 25 (21-29), projecting posteriorly. Coxigenital area with granules, with 4 annuli between coxae and genitalia, prosternal apodeme present forked; setae $1 b 8$ (7-9), 14 (13-14) apart; setae $1 a 25$ (24-27), 10 (9-12) apart; setae $2 a 32$ (29-40), 28 (28-33) apart. Leg I 35 (31-35), femur 10 (9-10), setae bv 10 (10-12); genua 5 (5-6), setae $l^{\prime \prime} 25$ (24-27); tibiae 8 (8-9), setae $l^{\prime} 8(6-8)$, setae located $1 / 4$ from dorsal base; tarsi 7 (6-7); empodia em simple 5 (4-5), 6-rayed, tarsi solenidia $\omega$ slightly tapered, 8 (7-8), setae $f t^{\prime} 17$ (17-18), setae $f t^{\prime \prime} 21$ (21-24), setae $u^{\prime}$ 2-3. Leg II 32 (31-33), femur 9 (8-10), setae bv 11 (10-12); genua 5 (4-5), setae $l^{\prime \prime} 10$ (10-12); 


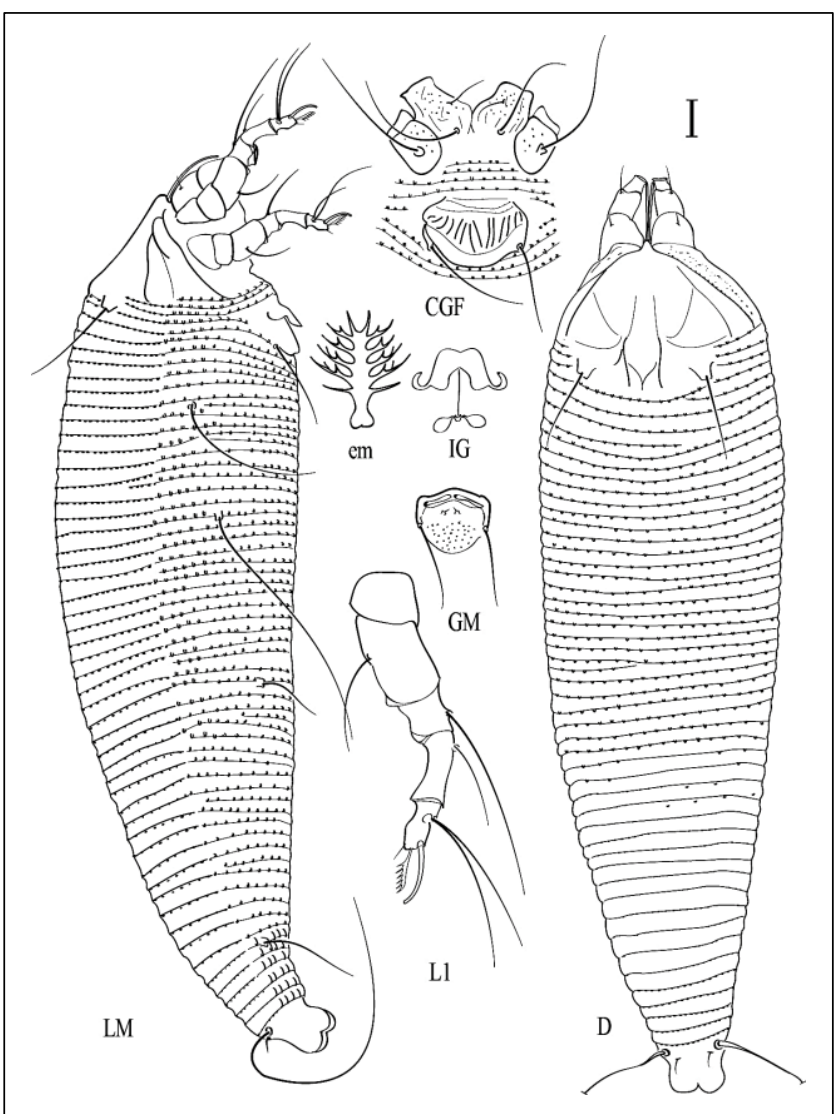

Fig. (5): Line drawings of Aceria malherbae Nuzzaci, 1985: D- Dorsal view of female; CGF- female coxigenital region; $\mathrm{GM}-$ male genitalia; IG- internal female genitalia; em- empodium; L1- Leg I; LM- lateral view of mite. Scale bars: $10 \mu \mathrm{m}$ for D, CGF, GM, IG, LM; $5 \mu \mathrm{m}$ for $\mathrm{L} 1 ; 2.5 \mu \mathrm{m}$ for em.
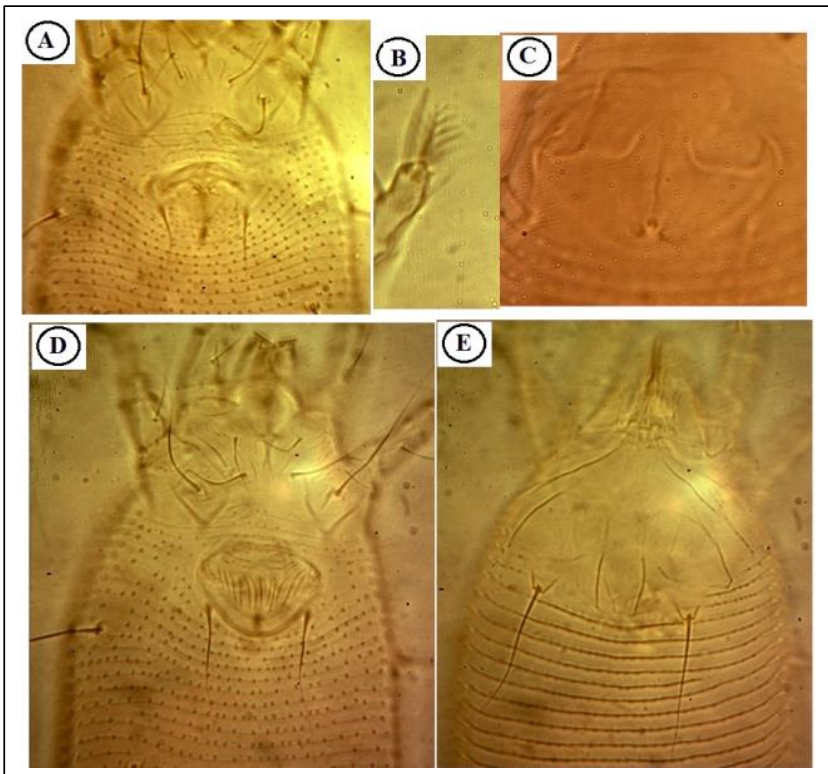

Fig. (7): Phase microphotograph of Aceria malherbae

Nuzzaci, 1985: A-male coxigenital region; B- empodium; C- internal female genitalia; D-female coxigenital region; E- prodorsal shield of female.

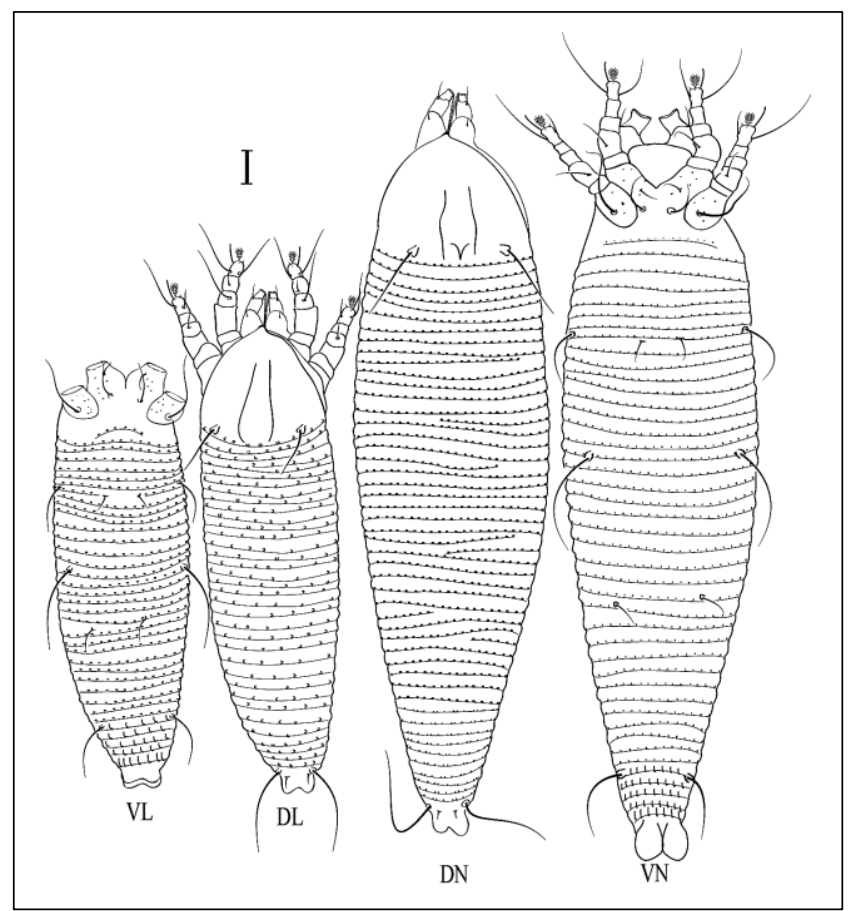

Fig. (6): Line drawings of Aceria malherbae Nuzzaci, 1985: DN- dorsal view of nymph; VN- ventral view of nymph; DL- dorsal view of larva; VL- ventral view of larva. Scale bars: $10 \mu \mathrm{m}$.

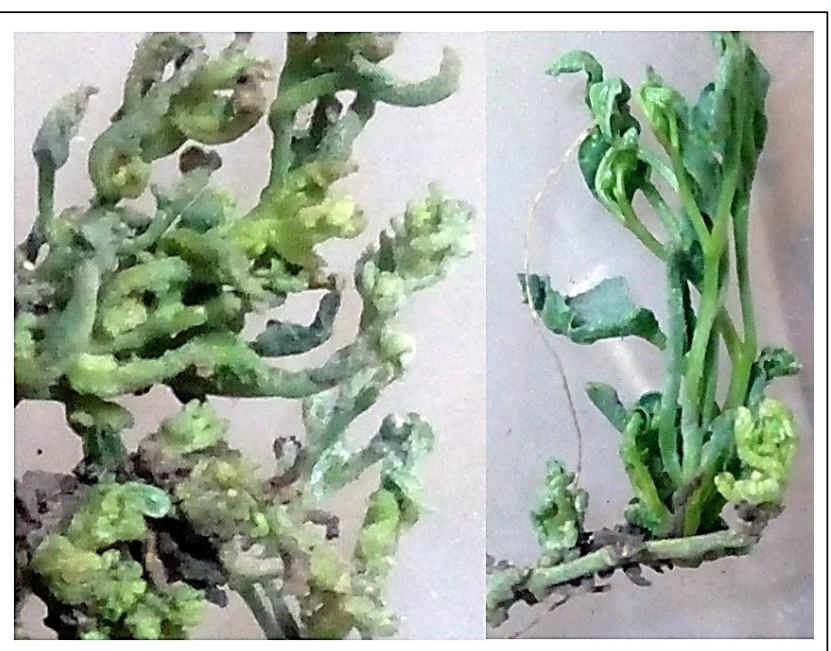

Fig. (8): Plant damage caused by the eriophyoid mite Aceria malherbae Nuzzaci, 1985. 
tibiae 7 (6-7); tarsi 7 (6-7); tarsal em simple 5 (4-5), 6-rayed, $\omega$ slightly tapered 8 (7-9), setae $f t^{\prime} 8(7-10)$, setae $f t^{\prime \prime} 21(20-24)$, tarsal setae $u^{\prime} 2-3$. Opisthosoma with 46 (45-48) dorsal semiannuli, with small round microtubercles on posterior annular margins, ventrally with 61 (60-63) semiannuli with round microtubercles on rear annular margins, the last $6^{\text {th }}$ ventral microtubercles liner. Lateral setae c2 35 (31-38), 63 (61-63) apart, on annulus 10 (9-10) from coxae II; setae $d 55$ (53-56), 53 (50-53) apart, on annulus 20 (19-20); setae e 12 (11-15), 28 (28-29) apart, on annulus 34 (33-35); setae $f 25$ (24-26), 29 (29-30) apart, on $6^{\text {th }}$ annulus from rear. Setae $h 265$ (64-67); setae hl 3 (3-4). External genitalia 18 (18-20), 28 (23-28) wide, coverflap with 12-14 longitudinal ridges, setae $3 a, 23$ (22-25), 24 (24-25) apart.

MALE. $(\mathrm{n}=10)$. Similar to female. Body vermiform, 150-160 excluding gnathosoma, 61-63 wide, 60-63 thick; whitish in life. Gnathosoma 23-25, chelicerae 16-17, setae ep 3-4, setae $d$ 5-6. Prodorsal shield shape and patterns similar to those of the female, 34-40 long, 45-50 wide; Scapular tubercles near the rear shield margin, 26-29 apart, setae sc 21-23, projecting diagonal posteriorly. Coxigenital area with granules, prosternal apodeme forked; setae $1 b$ 7-8, 12-1 apart; setae $1 a$ 14-16, 9-10 apart; setae $2 a$ 28-32, 24-25 apart. Leg I 29-30, femora 9-10, setae $b v$ 9-12; genu 4-5, setae $l^{\prime \prime} 15-17$; tibiae 6-7, setae l' 5-6; tarsi 7-8; tarsal empodia em simple 5-6, 6-rayed, tarsal solenidia $\omega$ distally slight tapered $7-8$, setae $f t^{\prime} 10-13$, setae $f t^{\prime \prime} 18-22$, setae $u^{\prime}$ 2-3.Leg II 28-30, femora 8-9, setae $b v$ 9-11;

genu 4-5, setae $l^{\prime \prime} 7-10$; tibiae 5-6; tarsi 5-6; tarsal empodia em simple 5-6, 6-rayed, $\omega$ slight tapered 5-6, setae $f t^{\prime}$ 5-8, setae $f t^{\prime \prime}$ 17-22, setae $u^{\prime} 2-3$. Opisthosoma with 44-46 dorsal semiannuli, ventrally with 50-55 semiannuli, microtubercles shape same that of the female. Lateral setae $c 228-32$, 60-61 apart, on annulus 9-10 from coxae II; setae $d$ 48-51, 43-44 apart, on annulus 18-19; setae $e$ 11-12, 25-26 apart, on annulus 30-31; setae $f 19-21,20-22$ apart, on $6^{\text {th }}$ annulus from rear. Setae h2 40-47; setae hl 2-3. External genitalia 17-18 long, 21-22 wide, with granules, setae $3 a$ 22-23, 17-18 apart.

Nymph (n=6). Body vermiform, 177-217; width 47-58. Gnathosoma 15-18, curved downward, setae $d$ 4-5, ep 2-3, chelicerae 14-16. Prodorsal shield semicircular, 38-42 long, 44-46 wide; median line indistinct on rear of prodorsal shield forming V-shaped marks, admedian lines incomplete and present only on the posterior half to two third of the prodorsal shield, submedian lines absent. Tubercles $s c$ on rear shield margin, 28-30 apart; sc 22-24.
Coxisternal plates With faint granules, $1 b 5-6$, 11-12 apart; $1 a$ 11-13, 8-9 apart; $2 a$ 21-23, 25-26 apart; 3a 6-7, 9-11 apart. Leg I 25-27; femur 7-8, bv 8-10; genu 4-5, $l^{\prime \prime} 12-15$; tibia 5-6, $l^{\prime} 4-5$; tarsus 4-5, ft' 11-15, ft" 15-20, setae $u^{\prime} 2$; solenidion $\omega$ 5-6; em 4, simple, 5-rayed. Leg II 23-26; femur 6-8, bv 8-10; genu 3-4, $l^{\prime \prime} 5-8$; tibia 4-5; tarsus 4-5, $f t^{\prime} 7-8, f t^{\prime \prime} 15-20$, setae $u^{\prime} 2 ; \omega$ 5-6; em 4-5, simple, 5-rayed. Opisthosoma With 47-49 dorsal semiannuli, with minute round microtubercles situated on rear margin of each semiannulus, 40-43 ventral annuli with minute round microtubercles, situated on rear margin of each semiannulus; elongated on the posterior annuli. Setae c2 18-20, 52-54 apart, on 8-9 ventral semiannulus; setae $d$ 30-33, 45-47 apart, on 17-18 ventral semiannulus; setae $e$ 9-10, 26-27 apart, on 26-27 ventral semiannulus; setae $f$ 20-22, 20-22 apart, on $5^{\text {th }}$ semiannulus from rear. Setae $h 12-3 ; h 225-28$.

LARVA ( $\mathrm{n}=4)$. Body vermiform, 115-146; width 41-4. Gnathosoma 15-18 curved downward, setae d 3-4, ep 1-2, chelicerae 12-15. Prodorsal shield semicircular, 35-37 long, 36-38 wide; median and submedian lines absent; admedian lines present on posterior $2 / 3$, subparallel. Tubercles $s c$ on rear shield margin, 22-24 apart; setae sc 14-17. Coxisternal plates With granules setae $1 b$ 5-6, 11-13 apart; 1 a 11-12, 9-10 apart; 2a 17-20, 25-28 apart. Setae $3 a$ 4-5, 10-11 apart. Leg I 24-26; femur 6-8, bv 5-6; genu 3-4, $l^{\prime \prime}$ 11-15; tibia 4-5, seta $l^{\prime} 3-4$; tarsus 5-6, ft' 11-15, $f^{\prime \prime}$ 14-17; $\omega$ 5-6; em 3-4, simple, 4-rayed. Leg II 22-24; femur 6-7, bv 5-6; genu 3, $l$ " 5-7; tibia 3-4; tarsus 5-6, ft' 4-5, $f t^{\prime \prime} 14-18 ; \omega$ 5-6; em 3-4, 4-rayed. Opisthosoma With subequal annuli, 33-36 annuli, with minute round microtubercles situated on rear margin of each semiannulus. Seta $c 213-15,35-37$ apart, on 7 ventral semiannulus; setae $d$ 25-27, 34-36 apart, on 15-16 ventral semiannulus; setae $e$ 7-8, 17-18 apart, on 20 ventral semiannulus; setae $f 16-18,22-23$ apart, on $5^{\text {th }}$ semiannulus from rear; $h 11-2 ; h 221-52$.

\section{Host plant from Egypt: Convolvulus arvensis L. (Convolvulaceae).}

Relation to host: Leaf folding, deformation of flowers and plants; the mites prevent plant reproduction; they cause bud swelling and injury.

Material examined. Fifty females, 15 males, 6 nymphs and 7 larvae on a microscope slide (slide no. EGPErio122.1-122.20), Kaha, Qalyubia

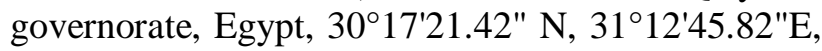
15 October 2015 and 15 September 2018 coll. Ashraf S. Elhalawany deposited in Fruit Trees Mites Dept., 
Collection, Plant Protection Research Institute (PPRI), Dokki, Egypt. Two slides in Plant Protection Research Institute collection. Two paratype slides are deposited at Plant Protection Research Institute collection.

Distribution: Canada; Greece; France; Iran; Italy; Mexico; South Africa; Spain; USA, Iran (de Lillo \& Amrine, personal communication).

Remarks: This is the first record of occurrence of females, males and immature stages of A. malherbae in Egypt. The holotype of female was described by Nuzzaci et al. 1985 from Italy; short description of the male and immature stages not found. Comparing the morphological characters of A. malherbae, as well as the original description given by Nuzzaci (1985), we did not find any regular differences between them. Morphology is similar, except for the length of the following characters: gnathosoma, body length, length and width of prodorsal shield, lengths of setae $c 2$ and $d$, and the number of genital coverflap. These setae are slightly shorter in mites from Italy than in mites from Egypt. Genital coverflap with 10 longitudinal striae from Italy (versus with 12-14 from Egypt).

\section{ACKNOWLEDGMENTS}

Thanks are expressed to Dr. Abdel Halim A. Mohamed (Flora and Phytotaxonomy Researches Department, Horticultural Research Institute, A. R.C., Dokki, Giza, Egypt) for identification of the host plant species.

\section{REFERENCES}

Abou-Awad, B.A. and El-Banhawy, E.M. 1992. New gall mites of the superfamily Eriophyoidea from East Africa (Arachnida: Acari). Acarologia, 33(1): 69-74.

Amrine, J.W. and Manson, D.C. 1996. Preparation, mounting and descriptive study of eriophyoid mites. In: Lindquist, E.E., Sabelis, M.W. \& Bruin J. (eds.), Eriophyoid mites: their biology, natural enemies and control. World Crop Pests, 6, Elsevier Science Publishing, Amsterdam, The Netherlands, pp. 383-396.

Amrine, J.W.Jr. and Stasny, T.A. 1994. Catalog of the Eriophyoidea (Acarina: Prostigmata) of the World. West Bloomfield, Michigan, USA, Indira Publishing House, 804 pp.

Amrine, J.W. Jr., Stasny, T.A. and Flechtmann, C.H.W. 2003. Revised keys to the world genera of the Eriophyoidea (Acari: Prostigmata). Indira Publishing House, West Bloomfield, Michigan, USA, 244 pp.
Baker, E.M. 2005. A new software for measuring leaf area, and area damaged by Tetranychus urticae Koch. J. Appl. Entomol., 129(3): 173-175. http://dx.doi.org/10.1111/j.40390418.2005.00948.x

Baker E.W., Kono T., Amrine J.W.Jr., DelfinadoBaker M. and Stasny T.A. 1996. Eriophyoid mites of the United States. Indira Publishing House, W. Bloomfield, MI. 394 pp.

Boczek, J. and Petanovic, R. U. 1994. Studies on eriophyoid mites (Acari: Eriophyoidea), XIV. Bull. Pol. Acad. Sci., Biol. Sci., 42(1): 87-93.

Boldt, P.E. and Sobhian, R. 1993. Release and establishment of Aceria malherbae (Acari: Eriophyidae) for control of field bindweed in Texas. Environ. Entomol., 22(1): 234-237. http://dx.doi.org/10.4039/Ent13151-4

Boulos, L. 1999. Flora of Egypt. Al-Hadara Publication, Cairo. 419 pp.

Craemer C. 1993. Eriophyidae (Acari) as potential control agents of South African weeds, with descriptions of a new species of Tegonotus Nalepa. M. Sc. Thesis, Rand Afrikaans University, Johannesburg, South Africa. 162 pp.

Craemer, C. 1995. Host specificity, and release in South Africa, of Aceria malherbae Nuzzaci (Acari, Eriophyoidea), a natural enemy of Convolvulus arvensis L. (Convolvulaceae). Afr. Entomol 3(2):213-215.

de Lillo, E. and Sobhian, R. 1996. A new Eriophyid species (Acari: Eriophyoidea) on Salsola spp. (Centrospermae, Chenopodiaceae) and a new report for Aceria tamaricis (Trotter). Entomologica, Bari, 30: 93-100. https://doi.org/10.15162/0425-1016/674

de Lillo, E., Craemer, C., Amrine, J.W.Jr. and Nuzzaci, G. 2010. Recommended procedures and techniques for morphological studies of Eriophyoidea (Acari: Prostigmata). Exp. Appl. Acarol., 51, 283-307.

Elhalawany, A.S. 2012. Survey of eriophyid mites on some fruit trees, with re-descriptions of two newly recorded species and a checklist of eriophyid mites in Egypt (Acari: Eriophyoidea). Egypt. Acad. J. Biolog. Sci., 5 (2): 205-216. http://dx.doi.org/10.21608/EABSA.2012.4833

Elhalawany, A.S. 2014a. Aculops awadi, a replacement species name for Aculops acaciae Abou-Awad\& Elsawi (Prostigmata Eriophyidae). Acarines, 8 (1): 67. http://dx.doi.org/10.21608/AJESA.2014.4913

Elhalawany, A. S. 2014 b. Two new species of eriophyoid mites (Prostigmata: Eriophyidae) infesting Acacia trees in Egypt. Acarines, 8 (2): 9-16.

Elhalawany, A.S. 2015. Description of one new species and two first records of eriophyid mites 
(Prostigmata: Eriophyidae) on grasses in Egypt. $5^{\text {th }}$ International Conference of Plant Protection Research Institute, 3-6 May 2015 Hurghada Egypt. Egypt J. Agric. Res., 93 (1a): 41-59.

Elhalawany, A.S. 2017. A new species in the genus Epitrimerus Nalepa (Acari: Eriophyidae) from Lantana camara L. in Egypt. $12^{\text {th }}$ Arab Congress of Plant Protection, Hurghada, Egypt, 4-10 November 2017. Egypt. Acad. J. Biolog. Sci., 10 (7): 149-160. http://dx.doi.org/10.21608/AJESA .2017 .1202

Elhalawany, A.S. and Ueckermann, E.A. 2015. Four new Aceria species (Acari: Trombidiformis: Eriophyidae) on Acacia nilotica from Egypt. International Journal of Acarology, 41(4): 272 282. http://dx.doi.org/10.1080/01647954.2015. 1035320

Elhalawany, A.S. and Ueckermann, E.A. 2018. Three new Aceria species (Acari, Trombidiformes, Eriophyidae) associated with the invasive weed Imperata cylindrica (L.) (Poaceae) from Egypt. International J. of Acarology, 44 (1): 7-20 https://doi.org/10.1080/01647954.2017.1402955

Holm, L., Pluckett, D.L., Ansho, J.V. and Herberger, J.P. 1977. The world's worst weeds. University Press of Hawaii, Honolulu. 609 pp.

Keifer, H.H. 1952. Eriophyid studies XIX. Bull. Calif. Dept. Agr., 41: 65-74.

Keifer H.H. 1962. Eriophyid studies B-8. Bur. Ent., Calif. Dept. Agric.: 1-20.

Keifer, H.H. 1966. Eriophyid studies B-21. Bur. Ent., Calif. Dept. Agric.: 1-24.

Kiedrowicz, A., Denizhan, E., Bromberek, K. Szydło1, W. and Skoracka, A. 2016. S Eriophyoid mites Acari Prostigmata: Eriophyoidea from Turkey Description of five new species. Zootaxa4066 (3): 255-270. http://doi.org /10.11646/zootaxa.4066.3.3

Lamb, K.P. 1953. New plant galls. II. Description of seven new species of gall mites and the galls which they cause. Trans. Roy. Soc. New Zealand, 80: 371-382.

Lindquist, E.E. 1996. External anatomy and notation of structures. In: Lindquist, E.E., Sabelis, M.W. \& Bruin, J. (eds.), Eriophyoid Mites: their Biology, Natural Enemies and Control. World Crop Pests, 6, Elsevier Science Publishing, Amsterdam, The Netherlands, pp. 3-31.

Lindquist, E.E., Sabelis, M.W. and Bruin, J. 1996. Eriophyoid mites their biology, natural enemies and control. Elsevier. World Crop Pests, 6, 822 pp.

Mohanasundaram, M. 1990. Studies on the genus Aceria (Acari: Eriophyidae) from south India. J. Acarol. 12(1 \& 2): 15-88.

Molliard, M. 1902. Caractères anatomiques de deux Phytoptocécidies caulinaires internes. Marcellia,
1(1/2): 21-29.

Nalepa A. 1899a. Neue Gallmilben. (18. Fort.) Anz. kais. Akad. Wiss., Math.-Nat. Kl., Wien 36(17): 217-218.

Nalepa A. 1899c. Neue Gallmilben. (19. Fort.) Anz. kais. Akad. Wiss., Math.-Natur Kl., Wien 36(19): 250.

Nalepa, A. 1900b. Neue Gallmilben. (20. Fort.) Anz. kais. Akad. Wiss., Math.-Nat, Kl., Wien 37(15): 154-156.

Nalepa, A. 1902a. Neue Gallmilben. (21 Fort.) Anz. kais. Akad. Wiss., Math.-Natur Kl., Wien 39(17): 221-223. (See recd. 2226)

Nuzzaci G., Mimmocchi T. and Clement S.L. 1985. A new species of Aceria (Acari: Eriophyidae) from Convolvulus arvensis L. (Convolvulaceae) with notes on other eriophyid associates of convolvulaceous plants. Entomologica, Bari, 20: 81-89. https://doi.org/10.15162/0425-1016/590

Rosenthal, S.S. 198. Field bindweed in Californiaextent and cost of infestation. California Agriculture, 7: 16-17.

Rosenthal, S.S. 1996. Biological control of weeds. Aceria, Epitrimerus and Aculus species and biological control of weeds. In: Eriophyoid Mites-Their Biology, Natural Enemies and Control. E.E. Lindquist, M.W. Sabelis and J. Bruin (Eds.), Elsevier Scinece. B.V. pp. 729-739.

Rosenthal, S.S. and Platts B.E. 1990. Host specificity of Aceria (Eriophyes) malherbae (Acari: Eriophyidae), a biological control agent for the weed, Convolvulus arvensis (Convolvulaceae). Entomophaga, 35(3): 459-463.

Roivainen, H. 1953. Some gall mites (Eriophyidae) from Spain. Arch. Inst. Aclim., 1: 9-43.

Skoracka, A., Smith, L., Oldfield, G., Cristofaro, M. and Amrine, J.W. Jr. 2010. Host-plant specificity and specialization in eriophyoid mites and their importance for the use of eriophyoid mites as biocontrol agents of weeds. Experimental and Applied Acarology 51(1-3): 93-113. http://doi.org/10.1007/s10493-009-9323-6

Tajaddod, S., Lotfollahi, P. and de-Lillo, E. 2018. Two new Aceria species (Acari: Trombidiformes: Eriophyoidea) from Ajabshir, Iran. Systematic \& Applied Acarology. 23(2): 305-313. http:// doi.org/10.11158/saa.23.2.7

Wilson, N. S. and Oldfield, G. N. 1966. New species of Eriophyid mites from Western North America with a discussion of Eriophyid mites on Populus. Ann. Entomol. Soc. Amer., 59(3): 585-599.

Xue, X.-F., Sadeghi, H. and Hong, X.-Y. 2009. Eriophyoid mites (Acari: Eriophyoidea) from Iran, with descriptions of three new species, one new record and a checklist. International Journal of 
Acarology, 35(6), 461-483. http://dx.doi.org/ 10.1080/01647950903427618

Xue, X.-F., Sadeghi, H., Hong, X.-Y. and Sinaie, S. 2013. New species and records of eriophyid mites from Iran (Acari: Eriophyidae). Systematic \& Applied Acarology, 18(1): 41-52. http:// dx.doi.org/10.11158/saa.18.1.5

Zaher, M.A. 1984. Survey and ecological studies on phytophagous, predaceous and soil mites in Egypt. 1. Phytophagous mites in Egypt (Nile Valley and Delta). PI 480 Programme U.S.A., Project No. EG-ARS-30, Grant No. FG-Eg-139, 228 pp.

Zaki, M.A. 2000. Identification and control of important weeds in Egypt. Al-Ahram Commercial Press - Kalyoub, Egypt, 266 pp. 